\title{
Growth factors contribute to the mediation of angiogenic capacity of glioma-associated mesenchymal stem cells
}

\author{
QING ZHANG $^{1,2}$, WEI XIANG ${ }^{1}$, BING-ZHOU XUE $^{1}$, DONG-YE YI $^{1}$, HONG-YANG ZHAO $^{1}$ and PENG FU ${ }^{1}$ \\ ${ }^{1}$ Department of Neurosurgery, Union Hospital, Tongji Medical College, Huazhong University of Science and Technology, \\ Wuhan, Hubei 430022; ${ }^{2}$ Brain Tumor Research Center, Beijing Neurosurgical Institute and Department of \\ Neurosurgery, Beijing Tiantan Hospital, Capital Medical University, Fengtai, Beijing 100070, P.R. China
}

Received May 14, 2020; Accepted October 19, 2020

DOI: 10.3892/ol.2021.12476

\begin{abstract}
Mesenchymal stem cells (MSCs) are important components of stromal cell populations and serve a crucial role in tumor growth and progression. Previously, our laboratory successfully isolated and cultured MSCs from human glioma issues and demonstrated that glioma-associated mesenchymal stem cells (gb-MSCs) participate in and maintain tumor angiogenesis. Furthermore, growth factors, such as fibroblast growth factor and vascular endothelial cell growth factor, were demonstrated to be associated with endothelial cell tube formation. However, the effect of transforming growth factor $\beta 1$ (TGF- $\beta 1$ ) and platelet-derived growth factor-BB (PDGF-BB) on the angiogenic activity of gb-MSCs remains unknown. The present study aimed therefore to explore their effects in gb-MSCs angiogenesis. In the present study, gb-MSCs were isolated from patients with glioma and were characterized using flow cytometry and differentiation experiments. Furthermore, the results from tube formation assay revealed that TGF- $\beta 1$ and PDGF-BB could mediate the angiogenic capacity of gb-MSCs in vitro. In addition, results from immunofluorescence demonstrated that gb-MSCs expressed TGF- $\beta 1 \mathrm{R}$ and PDGFR, which are the receptors for TGF- $\beta 1$ and PDGF-BB, respectively. Taken together, these findings indicated that TGF- $\beta 1$ and PDGF-BB may serve a crucial role in mediating gb-MSC angiogenesis, which might provide a
\end{abstract}

Correspondence to: Dr Peng Fu, Department of Neurosurgery, Union Hospital, Tongji Medical College, Huazhong University of Science and Technology, 1277 Jiefang Avenue, Wuhan, Hubei 430022, P.R. China

E-mail:pfu@hust.edu.cn

Abbreviations: gb-MSCs, glioma-associated mesenchymal stem cells; MSCs, mesenchymal stem cells; GBM, glioblastoma; TGF- $\beta 1$, transforming growth factor $\beta 1$; PDGF-BB, platelet-derived growth factor-BB; FGF, fibroblast growth factor; VEGF, vascular endothelial cell growth factor

Key words: glioma, transforming growth factor $\beta 1$, platelet-derived growth factor-BB, glioma-associated mesenchymal stem cells, angiogenic capacity, angiogenesis therapeutic strategy for targeting the angiogenic capacity of gb-MSCs in patients with glioma.

\section{Introduction}

Glioma, in particular glioblastoma (GBM), is the most immunosuppressive and lethal malignant brain tumor in adults, and accounts for $>30 \%$ of intracranial tumors current standard treatment options for malignant gliomas are multimodal and include surgical resection, postoperative radiotherapy and concomitant chemotherapy with temozolomide (1). Despite advancements in molecular understanding and therapies, the clinical benefits of glioma treatments remain limited (2-4), and the life expectancy of patients with GBM is only extended to $\sim 15$ months (5).

It has been reported that glioma-associated mesenchymal stem cells (gb-MSCs) express classical MSC surface markers, including CD105, CD73, CD90 and CD44, and lack the expression of CD14, CD34 and CD45. Furthermore, gb-MSCs can adhere to plastic and have the capacity to differentiate into osteoblasts, adipocytes and chondroblasts in vitro $(6,7)$. In addition, the percentage of gb-MSCs in high-grade glioma samples is closely related to survival of patients with GBM (8).

Vascular tube formation plays a crucial role in tumorigenesis (9). Human MSCs derived from bone marrow play a critical role in GBM-induced neovascularization (10). Previous studies from our laboratory demonstrated that gb-MSCs are considered as integral components involved in the pericyte transition and tumor vascular formation in the glioma microenvironment $(7,11)$. However, the involvement of glioma-secreted growth factors, such as transforming growth factor $\beta 1$ (TGF- $\beta 1$ ), platelet-derived growth factor-BB (PDGF-BB) and fibroblast growth factor 2 (FGF-2), in gb-MSC neovascularization and how they relate to vessel stabilization and contribute to malignancy, remain unclear. The present study aimed therefore to understand the role of growth factors, including PDGF-BB and TGF- $\beta 1$, in gb-MSC neovascularization. gb-MSCs may thus be considered as an anti-angiogenic treatment strategy in patients with GBM.

A better understanding of the molecular mechanism of gb-MSC angiogenesis is necessary for the development of a clinical tumor targeting strategy for glioma gene therapy. Certain cytokines, including vascular endothelial cell growth 
factor (VEGF), interleukin-6, interleukin-8, endothelial growth factors, TGF- $\beta 1$, FGF- 2 and PDGF-BB, are released from glioma cells and have been reported to mediate tumor angiogenesis $(12,13)$. Subsequently, the present study investigated the angiogenic capacity of TGF- $\beta 1$ and PDGF-BB on gb-MSCs.

\section{Materials and methods}

Isolation and culture of $g b$-MSCs. gb-MSCs were isolated from glioma samples that were collected from patients with GBM treated at the Neurosurgery Center in Wuhan Union Hospital and were processed within $1 \mathrm{~h}$. The age and sex of patients are not limited and the grade was III-IV. Briefly (7), glioma samples were washed three times using PBS to remove blood and impurities and were cut into 1-2 mm pieces. Tissue samples were placed into culture dishes and were digested using collagenase (Biyuntian Biotechnology Co., Ltd.) for $20 \mathrm{~min}$ at room temperature. Samples were filtered using a $70 \mu \mathrm{m}$ nylon mesh (Pall Life Sciences) and centrifuged at $350 \mathrm{x} \mathrm{g}$ for $10 \mathrm{~min}$ at room temperature. The mononuclear cells were collected following sample centrifugation through a Ficoll (2:1; Genview Corp.) density gradient at $350 \times \mathrm{g}$ for $20 \mathrm{~min}$ at $4^{\circ} \mathrm{C}$ Finally, cells were washed with PBS three times, cultured in DMEM (HyClone) supplemented with 10\% FBS (Biological Industries), $100 \mu / \mathrm{ml}$ penicillin and $100 \mu / \mathrm{ml}$ streptomycin (Gibco; Thermo Fisher Scientific, Inc.) and placed in a humidified atmosphere at $37^{\circ} \mathrm{C}$ with $5 \% \mathrm{CO}_{2}$.

Collection of conditioned medium. The human U87 glioblastoma cell line of unknown origin was purchased from the American Type Culture Collection (cat. no. HTB-14) and was identified by STR profiling. U87 and gb-MSCs were cultured in DMEM (HyClone; GE Healthcare Life Sciences) supplemented with 10\% FBS (Biological Industries) and $100 \mu / \mathrm{ml}$ penicillin and $100 \mu / \mathrm{ml}$ streptomycin (Gibco; Thermo Fisher Scientific, Inc.) in $25 \mathrm{~cm}^{2}$ culture flasks and placed in a humidified atmosphere at $37^{\circ} \mathrm{C}$ with $5 \% \mathrm{CO}_{2}$. When cells reached $50-60 \%$ confluence, they were washed three times with PBS and cultured in serum-free medium (0\% DMEM) for 3 days. Subsequently, the conditioned medium ( $0 \% \mathrm{gb}-\mathrm{CM}$, MSC-CM) was collected and centrifuged at 1,000 x $\mathrm{g}$ for $10 \mathrm{~min}$ at room temperature to remove cell debris. Aliquots of conditioned medium were stored at $-20^{\circ} \mathrm{C}$ until they further use (tube formation and ELISA assays).

Differentiation protocols. gb-MSCs were differentiated into osteocytes, adipocytes and chondrocytes following treatment with specific osteogenic, adipogenic or chondrogenic induction agents and maintenance media (all from StemCell Technologies, Inc.). These experiments were performed as previously described (7). Oil red $\mathrm{O}$ staining was used to examine adipogenic differentiation, alizarin red staining was used to investigate osteogenic differentiation and alcian blue staining was used to detect chondrogenic differentiation (all from Sigma-Aldrich; Merck KGaA). The standard medium was used as the control group.

Flow cytometric analysis. In order to characterize gb-MSCs, cells were digested using collagenase (Biyuntian Biotechnology
Co., Ltd.) at $37^{\circ} \mathrm{C}$, neutralized using complete media and finally centrifuged at $1,000 \mathrm{x}$ g for $5 \mathrm{~min}$ at room temperature. Supernatant was removed and the pellets were resuspended in fluorescent-activated cell sorting (FACS) buffer (Miltenyi Biotec $\mathrm{GmbH}$ ). These single-cell suspensions were incubated in the dark at $4^{\circ} \mathrm{C}$ for 30 min with FITC-, PE-Cy7-, APC-Cy7-, Percp- and APC-conjugated antibodies against human CD105, CD44, CD14, CD34 and CD31 (all from eBioscience; Thermo Fisher Scientific, Inc.). Subsequently, cells were centrifuged at $1,000 \mathrm{x}$ g for $5 \mathrm{~min}$ at room temperature, resuspended in PBS and analyzed using a FACS flow cytometer (BD Biosciences). The data were collected and analyzed using FlowJo V10 software (Tree Star, Inc.).

High-density micromass cultures of gb-MSCs. To investigate the chondrogenic differentiation of gb-MSCs in high-density pellet cultures (P3), complete medium containing a total of $2 \times 10^{5} \mathrm{gb}-\mathrm{MSCs}$ was placed in a $15 \mathrm{ml}$ polypropylene tube (Corning Inc.) and were centrifuged at $300 \mathrm{x}$ g for $10 \mathrm{~min}$. The cell pellets were collected and cultured in chondrogenic medium (Stemcell Technologies, Inc.) for 21 days, and half of the medium was changed during differentiation every three days. Cartilage nodules were formed by these cell pellets and they were subjected to standard paraffin embedding methods. The sections were then cut into $6 \mu \mathrm{m}$ slices and were subjected to hematoxylin-eosin staining (H\&E) staining, The slides were rinsed in PBS, incubated overnight at $4^{\circ} \mathrm{C}$ with anti-SOX-9 (1:100; cat. no. BS-4177R) and anti-collagen II (1:100; cat. no. 15943-1-AP) antibodies (Wuhan Sanying Biotechnology), and then incubated with an HRP-conjugated secondary antibody (1:1; cat. no. Ab7090; Wuhan Boster Biological Technology, Ltd.). Binding was detected using a DAB solution (Wuhan Boster Biological Technology, Ltd.). The tissues were counterstained using haematoxylin for $1 \mathrm{~min}$ at room temperature (Wuhan Boster Biological Technology, Ltd.). Images of the stained tissue samples were obtained using an Olympus light microscope (Olympus Corporation). For immunofluorescence, non-specific staining was blocked by pre-incubation with $5 \%$ goat serum (cat. no. AR1009) diluted in PBS for $30 \mathrm{~min}$ at room temperature. The primary antibodies used were as follows: goat anti-SOX-9 polyclonal antibody (1:200; Abcam; cat. no. ab185966) and rabbit anti-collagen II monoclonal antibody (1:100; Wuhan Boster Biological Technology, Ltd.; cat. no. ab3092). After incubation with the primary antibody overnight at $4^{\circ} \mathrm{C}$, sections were rinsed several times with PBS and incubated with the appropriate secondary antibodies at room temperature for $1 \mathrm{~h}$. The secondary antibodies used were as follows: Cy3-conjugated goat anti-rabbit (1:100; cat. no. BA1032) and FITC-conjugated goat anti-goat antibodies (1:100; Wuhan Boster Biological Technology, Ltd.; cat. no. BA1101). After washing with PBS, the sections were counterstained with DAPI (Beyotime Institute of Biotechnology) and mounted with anti-fade mounting medium. Immunofluorescence microscopy was performed with an Olympus light microscope (Olympus Corporation).

ELISA. ELISA kits (Neobioscience; cat. nos. EHC107b.96.10 and $\mathrm{EHC181.96)}$ were used to measure the levels of TGF- $\beta 1$ and PDGF-BB in the supernatants of gb-MSCs. All procedures were performed according to the manufacturers' instructions. 


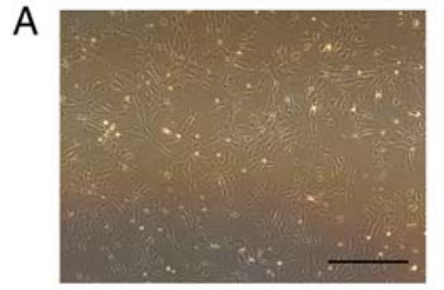

gb-MSC
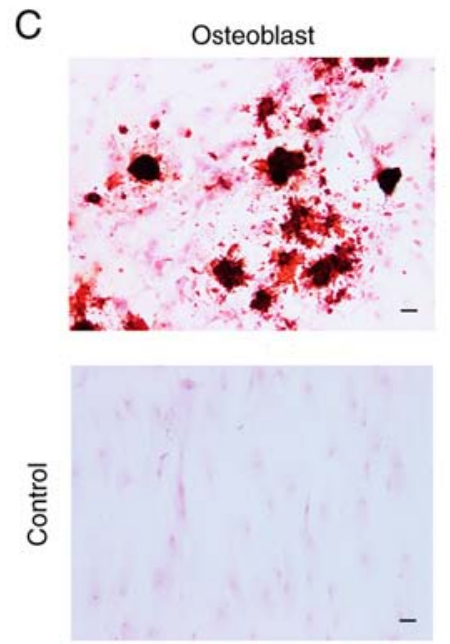

B
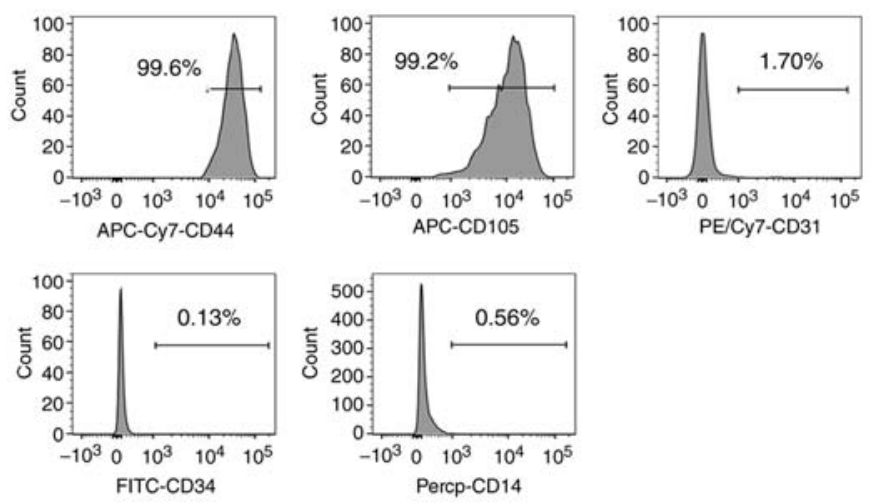

D

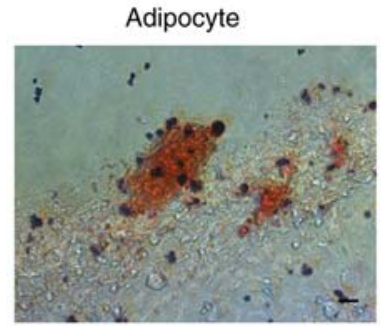

$\mathrm{E}$
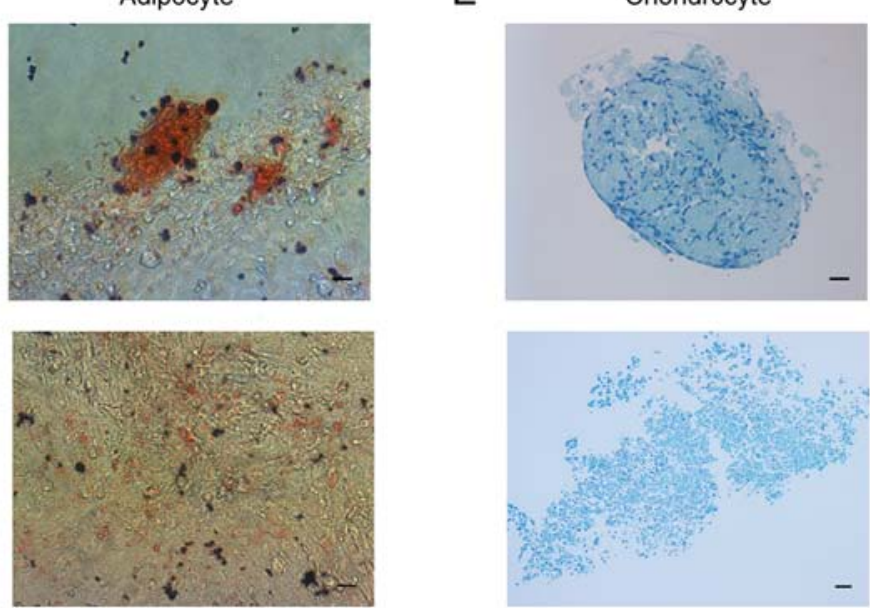

Figure 1. Characteristics of gb-MSCs. (A) Morphological features of gb-MSCs cultured in MSC medium (magnification, x40; scale bar, 200 $\mu$ m). (B) Flow cytometric results showed that gb-MSCs were positive for the MSC markers CD44 and CD105. Cells were negative for the cell markers CD31, CD34 and CD14. (C-E) Trilineage differentiation capacity of gb-MSCs. gbMSCs were treated in conditions specific for osteogenic differentiation, adipogenic differentiation and chondrogenic differentiation. Magnification, x200; scale bar, $50 \mu \mathrm{m}$. Lower panel shows staining of cells grown in MSC medium, which served as a control. gb-MSC, glioma-associated mesenchymal stem cells.

The absorbance was measured at $450 \mathrm{~nm}$ using a microplate reader. Each sample was assessed in triplicate.

Tube formation assay. Angiogenesis assays were performed according to consensus guidelines (14). Matrigel (BD Biosciences) was added to each well of flat-bottomed prechilled 96-well plates (Corning Inc.). After incubation at $37^{\circ} \mathrm{C}$ in $5 \% \mathrm{CO}_{2}$ for $40 \mathrm{~min}$, gb-MSCs were seeded $\left(1.5 \times 10^{5}\right.$ cells/well $)$ into the wells with serum-free glioma conditioned medium (0\% gb-CM), serum-free medium (0\% DMEM), serum-free medium containing rhTGF- $\beta 1$ or serum-free medium containing rhPDGF-BB, and plates were further incubated at $37^{\circ} \mathrm{C}$ in $5 \% \mathrm{CO}_{2}$. Each medium condition was assessed in triplicate. After $6 \mathrm{~h}$, tube formation was imaged using a light microscope (Olympus Corporation). Capillary-like tube formation was analyzed in three random fields of view per well using ImageJ software (National Institutes of Health).

Immunofluorescence. gb-MSCs (5,000 cells/well) were cultured in an eight-well chamber slide (Ibidi GmbH) at $37^{\circ} \mathrm{C}$. Once cells reached $70 \%$ confluence, they were washed with PBS three times, fixed with $4 \%$ paraformaldehyde for $15 \mathrm{~min}$ at room temperature, permeabilized with $0.5 \%$ Triton-X-100 for $20 \mathrm{~min}$ at room temperature and blocked with a solution containing donkey serum (Antgene) for $1 \mathrm{~h}$ at room temperature. Subsequently, cells were incubated with primary antibodies against PDGFR and TGF $\beta 1-R$ (R\&D Systems, Inc.) overnight at $4^{\circ} \mathrm{C}$. After being washed three times with PBS, cells were incubated with secondary antibodies labeled with rhodamine (Antgene) for $1 \mathrm{~h}$ at room temperature. In each experiment, non-specific staining by secondary antibodies was excluded by incubating a well without primary antibodies. Cells were also stained with DAPI for $5 \mathrm{~min}$ at room temperature (Antgene). Images were acquired using a fluorescent microscope (Olympus Corporation) and data were analyzed using Image-Pro Plus v6.0 (Media Cybernetics, Inc.).

Statistical analysis. Data were expressed as the means \pm standard deviation. Three independentexperiments were performed. Statistical analyses were performed using Graph-Pad Prism software 6 (GraphPad Software, Inc.). Kruskal-Wallis one-way ANOVA was used to compare variables followed by Dunnett's post hoc test if required. $\mathrm{P}<0.05$ was considered to indicate a statistically significant difference.

\section{Results}

gb-MSC stemness assessment by surface marker expression. gb-MSCs displayed a fibroblastic morphology consistent with that of MSCs (Fig. 1A). gb-MSCs were adherent in flasks 


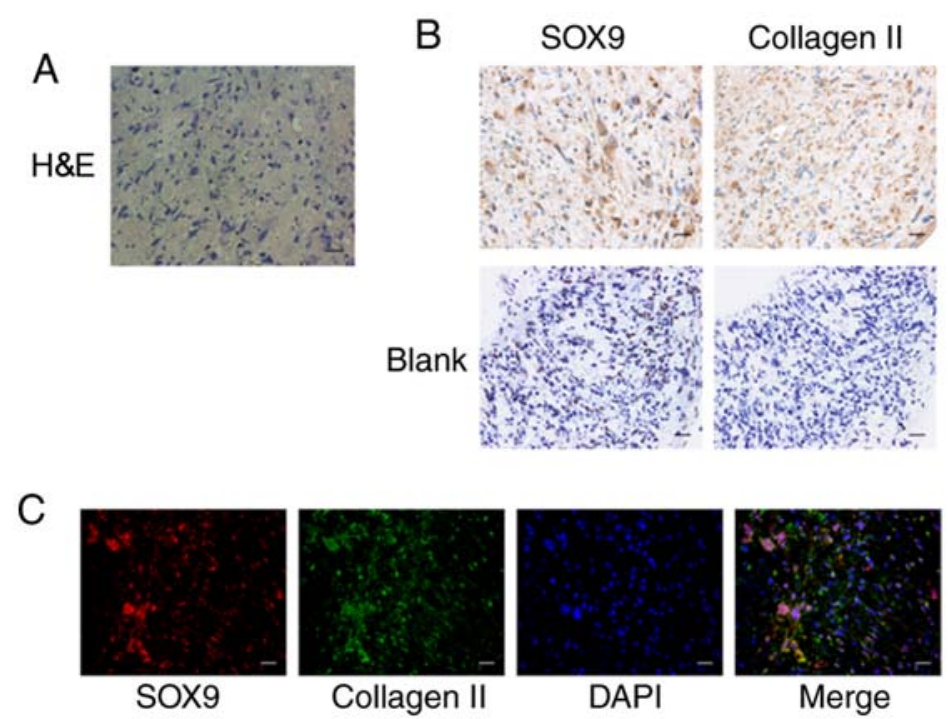

Figure 2. Chondrogenic differentiation of gb-MSCs in micromass cultured for 21 days. (A) H\&E staining of gb-MSCs. (B) SOX-9 and collagen II immunohistochemical staining of cartilage nodules. (C) Double staining for SOX-9 (red) and Collagen II (green) demonstrates that gb-MSCs have the capacity to differentiate into cartilage nodules. Magnification, x400; scale bar, $25 \mu \mathrm{m}$. gb-MSC, glioma-associated mesenchymal stem cells; H\&E, hematoxylin and eosin; SOX-9, SRY-Box transcription factor 9.

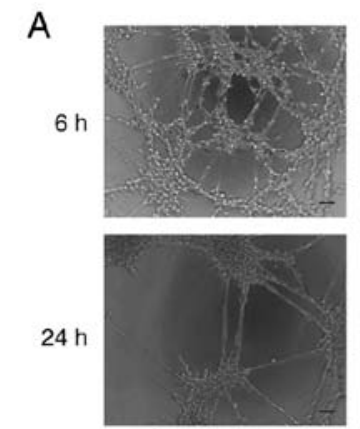

$0 \% \mathrm{gb}-\mathrm{CM}$
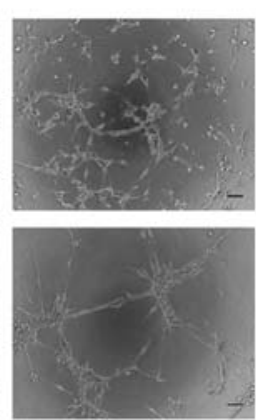

$0 \%$ DMEN

B

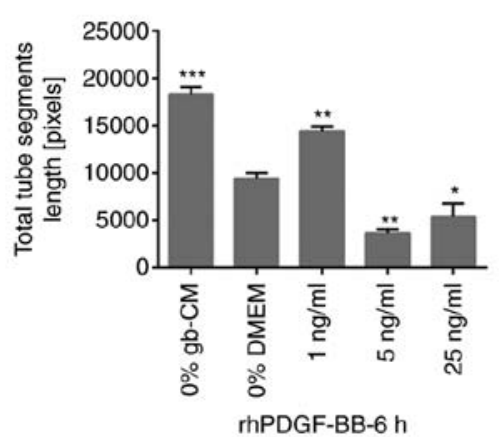

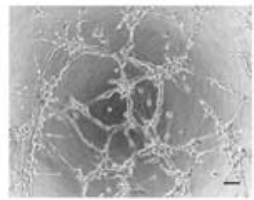

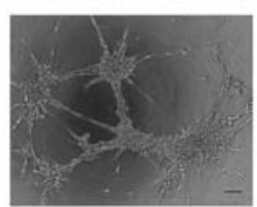

$1 \mathrm{ng} / \mathrm{ml}$
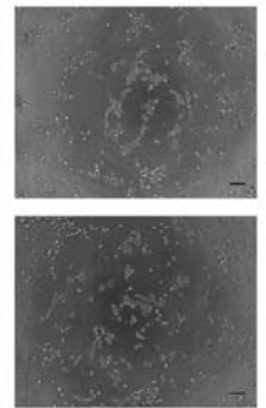

$5 \mathrm{ng} / \mathrm{ml}$
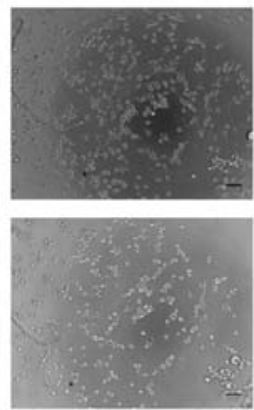

$25 \mathrm{ng} / \mathrm{ml}$

C

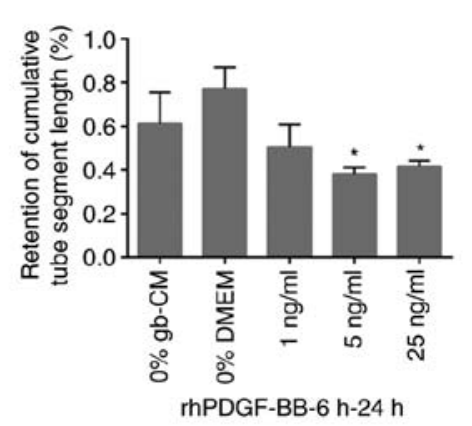

Figure 3. PDGF-BB mediated gb-MSC angiogenesis in vitro. (A) Tube formation in gb-MSCs cultured in different media for 6 h on Matrigel. (B, C) Total segment length and retention of tube segment length by gb-MSCs under different conditions. Magnification, $\mathrm{x} 100$; scale bar, $100 \mu \mathrm{m}$. ${ }^{*} \mathrm{P}<0.05$, ${ }^{* *} \mathrm{P}<0.01$ and ${ }^{* * *} \mathrm{P}<0.001$ vs. $0 \%$ DMEM group. $0 \%$ means that the medium contains no serum. gb-MSC, glioma-associated mesenchymal stem cells; CM, conditioned medium; PDFG-BB, platelet-derived growth factor-BB.

containing complete medium. To confirm the stemness of gb-MSCs, flow cytometry was used to evaluate the expression of certain molecular markers from passage 3 to passage 6 . The results demonstrated that gb-MSCs expressed CD44 and CD105, but did not express CD34, CD31 and CD14 (Fig. 1B).

Differentiation capacities of gb-MSCs. In vitro, gb-MSCs can differentiate into adipocytes, osteoblasts and chondrocytes under specific conditions, further promoting adipogenesis, osteogenesis and chondrogenesis (7), respectively. In the present study, the differentiation of the gb-MSCs into three linages was observed according to oil red $\mathrm{O}$ staining, alizarin red staining and alcian blue staining (Fig. 1C-E).

gb-MSCs can form cartilage structures in micromass culture. The chondrogenic differentiation ability of gb-MSCs was 


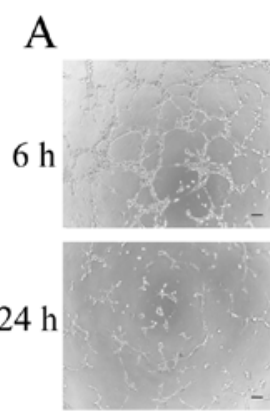

$0 \%$ gb-CM

B

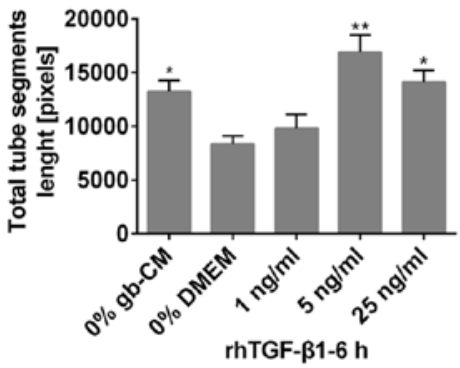

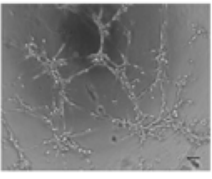
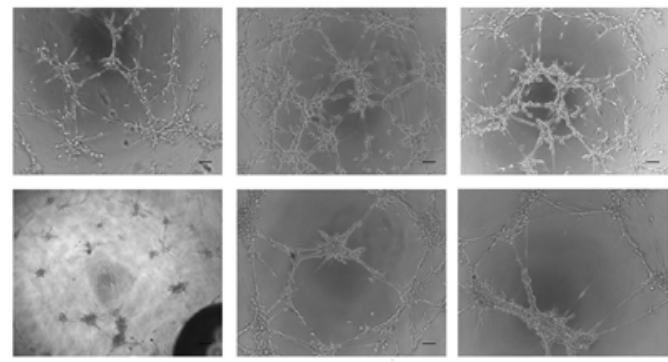

$1 \mathrm{ng} / \mathrm{ml}$

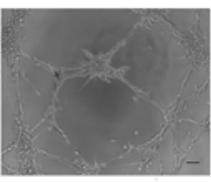

$5 \mathrm{ng} / \mathrm{ml}$

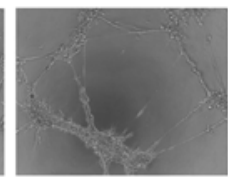

$25 \mathrm{ng} / \mathrm{ml}$

\section{$\mathrm{C}$}

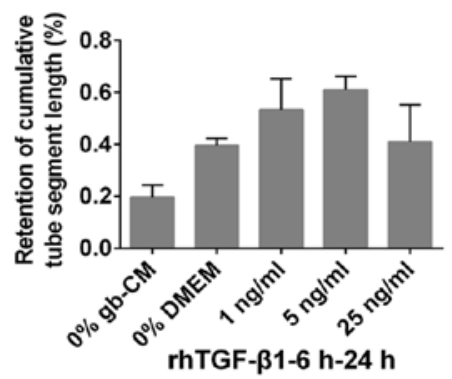

Figure 4. TGF- $\beta 1$ mediated gb-MSC angiogenesis in vitro. (A) Tube formation in gb-MSCs cultured in different media for 6 h on Matrigel. (B and C) Total segment length and retention of tube segment length by gb-MSCs under different conditions. Magnification, $\mathrm{x} 100$; scale bar, $100 \mu \mathrm{m}$. ${ }^{*} \mathrm{P}<0.05$ and ${ }^{* *} \mathrm{P}<0.01 \mathrm{vs}$. $0 \%$ DMEM group. $0 \%$ means that the medium contains no serum. gb-MSC, glioma-associated mesenchymal stem cells; CM, conditioned medium; TGF- $\beta 1$, transforming growth factor $\beta 1$.

A
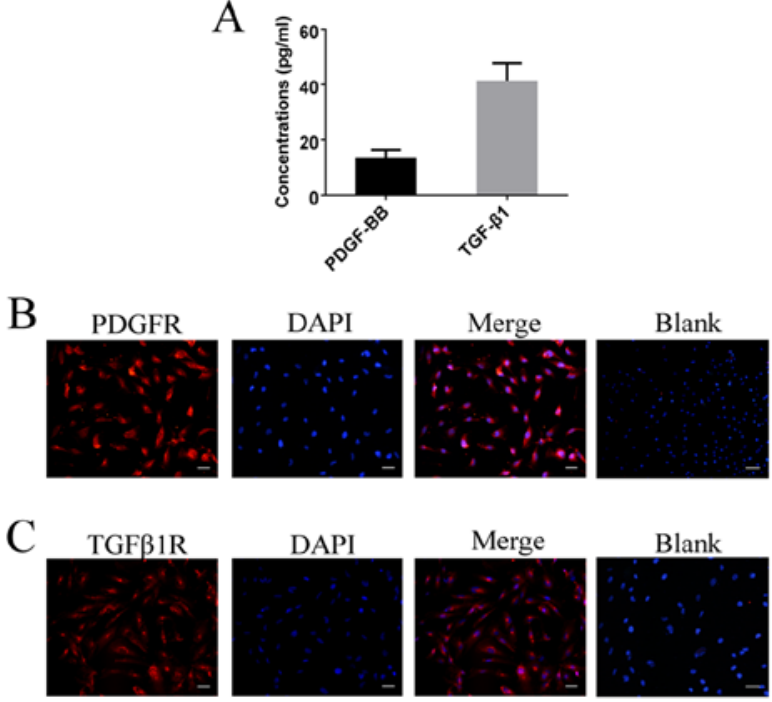

Figure 5. PDGFR and TGF $\beta 1 \mathrm{R}$ expression in gb-MSCs. (A) gb-MSCs secreted PDGF-BB and TGF- $\beta 1$. (B, C) gb-MSCs expressed PDGFR and TGF $\beta 1 R$. Magnification, $x 200$; scale bar=50 $\mu \mathrm{m}$. gb-MSC, glioma-associated mesenchymal stem cells; PDFG-BB, platelet-derived growth factor-BB; TGF- $\beta 1$, transforming growth factor $\beta 1$.

examined by high-density micromass cultures. This method determined whether cells purified from human glioma specimens could be used for cartilage tissue engineering applications. The results demonstrated that gb-MSC micromass cultures could promote the formation of proteoglycan (Figs. 1E and 2A). Results from immunohistochemistry staining demonstrated that collagen II (extracellular) and SOX-9 (nuclear) were highly expressed in micromass cultures compared with control (Fig. 2B). In addition, their coexpression in micromass cultures was confirmed (Fig. 2C).

$P D G F-B B$ and TGF- $\beta 1$ significantly improve the angiogenic capacity of gb-MSCs but decrease the stabilization of newly formed tubes. To examine whether TGF- $\beta 1$ and PDGF-BB could serve important roles in vascularization, an in vitro tube formation assay was performed using rhPDGF-BB and rhTGF- $\beta 1$. The results demonstrated a significant increase in gb-MSC vascularization when cells were treated with $1 \mathrm{ng} / \mathrm{ml}$ rhPDGF-BB after $6 \mathrm{~h}$ compared with 0\% DMEM group, whereas higher concentration of rhPDGF-BB (5 and $25 \mathrm{ng} / \mathrm{ml}$ ) did not allow gb-MSC vascularization (Fig. 3A and B). Furthermore, the results demonstrated that more tubes remained intact in the $0 \% \mathrm{gb}-\mathrm{CM}$ and \% DMEM treated chambers compared with those in the PDGF-BB (Fig. 3A and C).

Furthermore, the results demonstrated that rhTGF- $\beta 1$ could not increase significant tube formation at a lower concentration $(1 \mathrm{ng} / \mathrm{ml})$ after $6 \mathrm{~h}$, and the maximum vascularization of gb-MSCs was observed with a $5 \mathrm{ng} / \mathrm{ml}$ rhTGF- $\beta 1$ treatment (Fig. 4A and B). However, rhPDGF-BB (5 and $25 \mathrm{ng} / \mathrm{ml}$ ) was able to significantly decrease the stabilization of newly formed tubes compared with 0\% DMEM group from 6 to $24 \mathrm{~h}$ (Fig. 3A and C), and the tubes formed in cells treated with the lower concentration of rhPDGF-BB $(1 \mathrm{or} 5 \mathrm{ng} / \mathrm{ml})$ remained intact more efficiently than those in the 0\% DMEM-treated wells from 6 to $24 \mathrm{~h}$ (Fig. 4A and C).

gb-MSCs express PDGF-BB, TGF- $\beta 1, P D G F R$ and TGF- $\beta 1 R$. To determine the expression levels of PDGF-BB and TGF- $\beta 1$ in gb-MSCs, cell supernatant was collected and ELISA experiments were performed. The results demonstrated that 
gb-MSCs could secrete the growth factors PDGF-BB and TGF- $\beta 1$ (Fig. 5A). Furthermore, the expression of PDGFR and TGF $\beta 1 \mathrm{R}$ was examined in gb-MSCs using immunofluorescence (Fig. 5B and C).

\section{Discussion}

Previous studies have demonstrated that there are two subpopulations of gb-MSCs that serve different roles in glioma progression $(7,11)$. The robust angiogenic capacity of gb-MSCs could drive intracranial glioma development (7). A better understanding of the underlying mechanisms of gb-MSC angiogenesis is therefore crucial for the development of clinical treatments that target MSCs. The present study demonstrated that specific growth factors, TGF- $\beta 1$ and PDGF-BB, could mediate the angiogenesis of gb-MSCs in vitro. Previous studies from our laboratory reported some differences in the expression of long non-coding RNAs and miRNAs in gb-MSCs treated with different conditioned media. Furthermore, predicted target genes were enriched for genes involved in angiogenesis $(7,11)$. This may suggest that the related angiogenesis genes were upregulated to enhance the expression of PDGF-BB and TGF- $\beta 1$, further promoting angiogenic capacity by specific signaling pathways.

TGF- $\beta 1$, a member of the cytokine family that is important for mediating the malignant phenotype of human brain gliomas, is secreted by malignant gliomas in vitro and in vivo $(15,16)$. TGF- $\beta 1$ expression is positively correlated with higher-grade gliomas and with the induction of angiogenesis (17). In addition, TGF- $\beta 1$ plays a significant role in glioma angiogenesis in vivo $(18,19)$. A previous study reported that TGF- $\beta 1$ might be considered as a vital cytokine in MSC angiogenesis (11). The results from the present study demonstrated that TGF $\beta 1 \mathrm{R}$ is expressed in gb-MSCs and that gb-MSC angiogenesis was increased in response to TGF- $\beta 1$. Therefore, TGF- $\beta 1$ may serve a crucial role in gb-MSC angiogenic capacity. These findings were consistent with data from a recent study demonstrated that TGF- $\beta 1$ secreted by primary malignant glioma can stimulate angiogenesis of MSCs (11).

The cytokine PDGF-BB, which binds to its receptor PDGFR, plays a unique role in the regulation of angiogenesis $(20,21)$. Previous studies have demonstrated that PDGF-BB contributes to angiogenesis in tumor tissues $(21,22)$. Our laboratory reported that PDGF-BB is secreted by glioma cells (10) and serves a crucial role in the regulation of MSC angiogenic capacity. It has also been demonstrated that PDGF-BB/PDGF $\beta R$ interaction plays a functional role in tumor angiogenesis (20). In the present study, the expression of PDGFR in gb-MSCs was assessed, and gb-MSC angiogenic capacity in response to PDGF-BB was determined. The results demonstrated that PDGF-BB played an important role in stimulating the angiogenic capacity of gb-MSCs. These findings were consistent with data from a previous study demonstrating that TGF- $\beta 1$ can stimulate MSC angiogenesis (10). Taken together, these results suggested that TGF- $\beta 1$ and PDGF-BB may contribute to the modulation of gb-MSC angiogenesis.

In conclusion, the present study successfully isolated gb-MSCs from human glioma tissues and demonstrated that their fibroblast-like morphology and surface markers were similar to those of classic MSCs. In addition, the results demonstrated that TGF $\beta 1$ and PDGF-BB could improve the angiogenic capacity of gb-MSCs and promote the expression of the growth factor receptors TGF $\beta$-R1 and PDGF $\beta R$. Further investigation of the underlying mechanisms of gb-MSC angiogenesis may provide novel insights into targeting vessel formation to treat patients with glioma.

\section{Acknowledgements}

Not applicable.

\section{Funding}

The present study was supported by the National Natural Science Foundation of China (grant no. 81572488).

\section{Availability of data and material}

All data generated or analyzed during this study are included in this published article.

\section{Authors' contributions}

QZ and WX designed the study, analyzed the data and drafted the manuscript. BX, DY and HZ collected and sorted the data and analyzed the related literature PF, managed the project, generated the outline of the manuscript and revised the language. All authors read and approved the final version.

\section{Ethics approval and consent to participate}

The present study was approved by The Ethical Committee of Tongji Medical College of Huazhong University of Science and Technology (Wuhan, China; approval no. S207). Written informed consent was obtained from all patients and the experiments were conducted in accordance with the Declaration of Helsinki.

\section{Patient consent for publication}

Not applicable.

\section{Competing interests}

The authors declare that they have no competing interests.

\section{References}

1. Stupp R, Mason WP, van den Bent MJ, Weller M, Fisher B, Taphoorn MJ, Belanger K, Brandes AA, Marosi C, Bogdahn U, et al: Radiotherapy plus concomitant and adjuvant temozolomide for glioblastoma. N Engl J Med 352: 987-996, 2005.

2. Fu P, He YS, Huang Q, Ding T, Cen YC, Zhao HY and Wei X: Bevacizumab treatment for newly diagnosed glioblastoma: Systematic review and meta-analysis of clinical trials. Mol Clin Oncol 4: 833-838, 2016.

3. Holland EC: Glioblastoma multiforme: The terminator. Proc Natl Acad Sci USA 97: 6242-6244, 2000.

4. Surawicz TS, Davis F, Freels S, Laws ER Jr and Menck HR: Brain tumor survival: Results from the national cancer data base. J Neurooncol 40: 151-160, 1998.

5. DeAngelis LM: Brain tumors. N Engl J Med 344: 114-123, 2001. 
6. Hossain A, Gumin J, Gao F, Figueroa J, Shinojima N, Takezaki T, Priebe W, Villarreal D, Kang SG, Joyce C, et al: Mesenchymal stem cells isolated from human gliomas increase proliferation and maintain stemness of glioma stem cells through the IL-6/gp130/STAT3 pathway. Stem Cells 33: 2400-2415, 2015

7. Zhang Q, Yi DY, Xue BZ, Wen WW, Lu YP, Abdelmaksou A Sun MX, Yuan DT, Zhao HY, Xiong NX, et al: CD90 determined two subpopulations of glioma-associated mesenchymal stem cells with different roles in tumour progression. Cell Death Dis 9: 1101, 2018.

8. Shahar T, Rozovski U, Hess KR, Hossain A, Gumin J, Gao F, Fuller GN, Goodman L, Sulman EP and Lang FF: Percentage of mesenchymal stem cells in high-grade glioma tumor samples correlates with patient survival. Neuro Oncol 19: 660-668, 2017.

9. Birnbaum T, Roider J, Schankin CJ, Padovan CS, Schichor C, Goldbrunner R and Straube A: Malignant gliomas actively recruit bone marrow stromal cells by secreting angiogenic cytokines. J Neurooncol 83: 241-247, 2007.

10. Birnbaum T, Hildebrandt J, Nuebling G, Sostak P and Straube A Glioblastoma-dependent differentiation and angiogenic potential of human mesenchymal stem cells in vitro. J Neurooncol 105: 57-65, 2011.

11. Yi D, Xiang W, Zhang Q, Cen Y, Su Q, Zhang F, Lu Y, Zhao H and Fu P: Human glioblastoma-derived mesenchymal stem cell to pericytes transition and angiogenic capacity in glioblastoma microenvironment. Cell Physiol Biochem 46: 279-290, 2018.

12. Kargiotis O, Rao JS and Kyritsis AP: Mechanisms of angiogenesis in gliomas. J Neurooncol 78: 281-293, 2006.

13. Keerl S, Gehmert S, Gehmert S, Song YH and Alt E: PDGF and bFGF modulate tube formation in adipose tissue-derived stem cells. Ann Plast Surg 64: 487-490, 2010.

14. Frei K, Gramatzki D, Tritschler I, Schroeder JJ, Espinoza L, Rushing EJ and Weller M: Transforming growth factor- $\beta$ pathway activity in glioblastoma. Oncotarget 6: 5963-5977, 2015.

15. Liubich LD, Kovalevska L, Lisyany MI, Semenova VM, Malysheva TA, Stayno LP and Vaslovych VV: TGF- $\beta 1$ expression by glioma C6 cells in vitro. Exp Oncol 39: 258-263, 2017.
16. Ferrari G, Cook BD, Terushkin V, Pintucci G and Mignatti P: Transforming growth factor-beta 1 (TGF-beta1) induces angiogenesis through vascular endothelial growth factor (VEGF)-mediated apoptosis. J Cell Physiol 219: 449-458, 2009.

17. Kaminska B, Kocyk M and Kijewska M: TGF beta signaling and its role in glioma pathogenesis. Adv Exp Med Biol 986: 171-187, 2013.

18. Yang XJ, Chen GL, Yu SC, Xu C, Xin YH, Li TT, Shi Y, Gu A, Duan JJ, Qian C, et al: TGF-beta1 enhances tumor-induced angiogenesis via JNK pathway and macrophage infiltration in an improved zebrafish embryo/xenograft glioma model. Int Immunopharmacol 15: 191-198, 2013.

19. Battegay EJ, Rupp J, Iruela-Arispe L, Sage EH and Pech M: PDGF-BB modulates endothelial proliferation and angiogenesis in vitro via PDGF beta-receptors. J Cell Biol 125: 917-928, 1994.

20. Cumpanas AA, Cimpean AM, Ferician O, Ceausu RA, Sarb S, Barbos V, Dema A and Raica M: The involvement of PDGF-B/PDGFR $\beta$ axis in the resistance to antiangiogenic and antivascular therapy in renal cancer. Anticancer Res 36: 2291-2295, 2016.

21. Xiong B, Gong LL, Zhang F, Hu MB and Yuan HY: TGF beta1 expression and angiogenesis in colorectal cancer tissue. World J Gastroenterol 8: 496-498, 2002.

22. Xue Y, Lim S, Yang Y, Wang Z, Jensen LD, Hedlund EM, Andersson P, Sasahara M, Larsson O, Galter D, et al: PDGF-BB modulates hematopoiesis and tumor angiogenesis by inducing erythropoietin production in stromal cells. Nat Med 18: 100-110, 2011.

This work is licensed under a Creative Commons Attribution-NonCommercial-NoDerivatives 4.0 International (CC BY-NC-ND 4.0) License. 